myoclonic seizures not related to sleep. EEG showed burst suppression pattern, refractory to medication and typical of Ohtahara syndrome. Seizures were unresponsive to pyridoxal phosphate and AEDs. Finally, folinic acid $5 \mathrm{mg} / \mathrm{kg}$ per day was added with dramatic response. The child was seizure-free in 1 day, and the EEG showing only generalized slowing had no burst-suppression pattern 4 days later. She was seizure-free for 6 months but relapsed at 10 months of age during a febrile illness. Seizures were finally controlled with sodium valproate and clobazam, but she had severe global developmental delay. Mutation analysis of the ALDH7A1 (antiquitin) gene was negative. Mutation screening revealed a missense mutation in exon 16 of the STXBP1 gene. Analysis of parental DNA confirmed the mutation as de novo. (Tso WWY, Kwong AKY, Fung CW, Wong VCN. Folinic acid responsive epilepsy in Ohtahara syndrome caused by STXBP1 mutation. Pediatr Neurol 2014 Feb;50(2):177-80).

COMMENTARY. In addition to Ohtahara syndrome, STXBP1 mutations are associated with West syndrome, and learning disabilities. For Ohtahara syndrome caused by STXBP1 mutations, a trial of folinic acid is indicated. Folinic acid responsive seizures are identical to pyridoxine-dependent epilepsy, and both are caused by a-AASA dehydrogenase deficiency with mutations in the ALDH7A1 (antiquitin) gene [1]. Two patients with neonatal epileptic encephalopathy are reported whose CSF showed the marker of folinic acid-responsive seizures, but who responded to pyridoxine [1]. Treatment with both pyridoxine and folinic acid is recommended for infants with alphaAASA dehydrogenase deficiency. The Hong Kong patient's seizures caused by mutations in the STXBP1 gene showed transient folinic acid responsiveness and no response to pyridoxine.

\title{
References.
}

1. Gallagher RC, et al. Ann Neurol. 2009 May;65(5):550-6.

\section{EARLY NEUROIMAGING IN MOLYBDENUM COFACTOR DEFICIENCY}

Investigators at Wakayama Medical University, Japan, report the neuroimaging features soon after birth in 2 siblings with molybdenum cofactor deficiency (MoCoD) type A. Seizures occurred soon after birth. Brain ultrasound revealed subcortical multicystic lesions in the frontal white matter, and brain MRI at 4-24 hours after birth showed restricted diffusion on diffusion-weighted images, with severe atrophy of the entire cortex within 1 month. The corpus callosum was absent or underdeveloped in both infants. (Higuchi R, Sugimoto T, Tamura A, et al. Early features in neuroimaging of two siblings with molybdenum cofactor deficiency. Pediatrics 2014 Jan;133(1):e267-71).

COMMENTARY. MoCo is a coenzyme common to sulfite oxidase, xanthine dehydrogenase and aldehyde oxidase. Encephalopathy in MoCoD may result from isolated sulfite oxidase deficiency. MoCoD presents with intractable seizures in the neonatal period and MRI findings are similar to those of hypoxic ischemic encephalopathy (HIE). Since MoCoD progresses rapidly after birth, early diagnosis suspected by MRI findings can be confirmed with low plasma uric acid, positive sulfite 
dipstick in fresh urine, and elevated urine and plasma s-sulfocysteine. In infants with HIE these markers are absent and the plasma uric acid is elevated. On diffusion weighted imaging within 1 week after birth, patients with HIE show an increased signal in all cortical and subcortical areas, whereas in patients with $\mathrm{MoCoD}$ these findings are not uniform.

In addition to HIE, infants with $\mathrm{MoCoD}$ may present with neonatal hyperekplexia, unresponsive to clonazepam [1], and as pyridoxine-dependent epilepsy [2]. Two siblings with pyridoxine-responsive seizures and increased urinary excretion of a-AASA were diagnosed with MoCoD and a mutation in the MOCS2 gene. A trial of pyridoxine is recommended in patients with MoCo or sulfite oxidase deficiencies [2].

\section{References.}

1. Macaya A, et al. Neuropediatrics. 2005 Dec;36(6):389-94.

2. Struys EA, et al. Pediatrics. 2012 Dec;130(6):e1716-9.

\section{DEGENERATIVE DISEASES}

\section{ALZHEIMER GENE LINKED TO BRAIN DEVELOPMENT}

Investigators at Brown University, Providence, RI, and other imaging genetic centers in the US, compare MRI measurements of white matter myelin water fraction (MWF) and gray matter volume (GMV) in healthy infant carriers and noncarriers of the apolipoprotein E (APOE) e4 allele, the major susceptibility gene for late-onset Alzheimer disease (AD). Infant e4 carriers, ages 2-25 months, had lower MWF and GMV measurements than noncarriers in precuneus, posterior/middle cingulate, lateral temporal, and medial occipitotemporal regions, areas affected by $\mathrm{AD}$, whereas these measures were greater in frontal regions, and an attenuated relationship between MWF and age was evident in posterior white matter regions. The study demonstrates some of the earliest brain changes associated with a genetic predisposition to $\mathrm{AD}$, and the role of APOE in normal human brain development and AD pathology. (Dean DC $3^{\text {rd }}$, Jerskey BA, Chen $\mathrm{K}$, et al. Brain differences in infants at differential genetic risk for late-onset Alzheimer disease: a cross-sectional imaging study. JAMA Neurol 2014 Jan 1;71(1):11-22).

COMMENTARY. In a comment (Alzheimer gene APOE e4 linked to brain development in infants), Drs McDonald and Krainc of Northwestern University Feinberg School of Medicine find that this study highlights compelling evidence of the influence of the APOE e4 allele on brain structure in young infants. It remains to be determined whether these neurodevelopmental observations specifically influence AD pathogenesis in later life [1]. In an editorial, Growdon JH, and Hyman BT allude to data emphasizing effects of B-amyloid on neural plasticity during brain development, a peptide elevated in Down syndrome where trisomy 21 leads to an extra copy of the amyloid precursor protein and early onset AZ [2].

A study of effect of age and APOE genotype on neuropathological changes in Down syndrome hippocampal formation found that individuals who had inherited the APOE e4 genotype contained more than twice the amyloid burden of non-carriers. The level of amyloid deposition in Down syndrome patients is higher than in sporadic AZ 